\title{
Casimir dark energy, stabilization of the extra dimensions and Gauss-Bonnet term
}

\author{
Pitayuth Wongjun ${ }^{\mathrm{a}}$ \\ The Institute for Fundamental Study, Naresuan University, Phitsanulok 65000, Thailand
}

Received: 21 June 2014 / Accepted: 17 December 2014 / Published online: 14 January 2015

(C) The Author(s) 2015. This article is published with open access at Springerlink.com

\begin{abstract}
A Casimir dark energy model in a five-dimensional and a six-dimensional spacetime including nonrelativistic matter and a Gauss-Bonnet term is investigated. The Casimir energy can play the role of dark energy to drive the late-time acceleration of the universe while the radius of the extra dimensions can be stabilized. The qualitative analysis in four-dimensional spacetime shows that the contribution from the Gauss-Bonnet term will effectively slow down the radion field at the matter-dominated or radiationdominated epochs so that it does not pass the point at which the minimum of the potential will arise before the minimum has formed. The field then is trapped at the minimum of the potential after the formation leading to the stabilization of the extra dimensions.
\end{abstract}

\section{Introduction}

The late-time acceleration of the universe was discovered by observing the behavior of the supernovae of type Ia (SN Ia) $[1,2]$. Recent observations imply that about $72 \%$ of the energy density of the universe consists of an unknown constituent called "dark energy" [3-5]. One of the simple candidates which is able to fit with the current observational data is the cosmological constant. Theoretically, the cosmological constant can be interpreted as vacuum energy. It is found that the energy scale of the vacuum energy calculated from particle physics theory is far larger than the observed value of the cosmological constant [6]. It is also plagued by the coincidence problem, which amounts to the fact that the energy densities of cosmological constant and dark matter are significantly different throughout the history of the universe, while their energy densities are of the same order at the present time [7-10]. Therefore, various kinds of dynamical models for dark energy were proposed in order to explain the late-time acceleration of the universe $[11,12]$, for exam-

\footnotetext{
a e-mail: pitbaa@gmail.com
}

ple, quintessence models [13,14], k-essence models [15-17], Galilean models [18] and their generalization [19,20], vector field models [21,22], three-form field models [23,24] and holographic dark energy models [25]. Moreover, there are many modified gravity models constructed in order to explain this late-time acceleration of the universe, for example, $f(R)$ gravity models $[26,27]$ (for viable cosmological models, see Refs. [28-30]), $f(G)$ gravity models [31] and, recently, massive gravity models $[32,33]$. Among the various dark energy models, there is a model, motivated from fundamental theories such as string theory, called the "Casimir dark energy model" $[34,35]$. We will focus on this model in the present work.

A fundamental theoretical framework that may be able to provide a description of the late-time acceleration of the universe is offered by string theory. Generally, string theory requires the presence of extra dimensions. However, from the observation point of view, we live in four-dimensional spacetime. This implies that the extra dimensions have to be compactified. It is not easy to obtain a mechanism for stabilizing the extra dimensions, while providing a viable model of dark energy [36-38] and the lack of such a mechanism is often called the "moduli stabilization problem". However, searches for a viable moduli stabilization mechanism are ongoing; for example see $[39,40]$.

One of the most promising dark energy models that provides a solution for the moduli stabilization problem is the Casimir dark energy model $[34,35,41]$. The Casimir energy is a vacuum energy emerging from imposing boundary conditions on the quantum fluctuations of fields. The Casimir energy is very tiny compared to the vacuum energy calculated from particle physics theory. It is worthwhile to interpret the cosmological constant as the Casimir energy instead of the vacuum energy since the Casimir energy can naturally emerge from the compactification mechanism. Moreover, this dark energy candidate also provides the mechanism for stabilizing the extra dimensions automatically. However, in order to compare the results of the model to 
the standard history of the universe, we need to include nonrelativistic matter. Unfortunately, adding the non-relativistic matter to the model destroys the stabilization mechanism of the extra dimensions $[35,42]$. For the effective theory in four-dimensional spacetime, the size of the extra dimension will be characterized by a scalar field, the so-called moduli field or radion field, and the Casimir energy plays the role of the potential of the moduli field. The dynamics of the moduli field can be analyzed by using the potential in effective theory in four-dimensional spacetime. The qualitative analysis shows that the minimum of the potential of the moduli field will disappear and its slope will increase when non-relativistic matter is included. Hence, the moduli or radion field will rapidly roll down and then pass away from the minimum point before it has formed, which eventually leads to the destabilization of the extra dimensions. In order to restore the stabilization mechanism, a modified Casimir dark energy model in which the aether field is added has been investigated [42]. The effects of the aether field in the higherdimensional spacetime were also investigated in $[43,44]$ and a key result in four-dimensional spacetime is that the slope of the potential is decreased. In other words, the force acting on the radion field during the matter-dominated period is reduced. Thus the radion field slowly rolls down during the matter-dominated period and it has enough time for waiting for the formation of the potential minimum at the late time. This eventually leads to the stabilization of the extra dimensions. Unfortunately, the aether field which can provide this viable model is plagued by ghost instabilities implying an unstable theoretical model [45-48]. It was also found that the linear perturbations in the cosmological background of the aether model grow exponentially.

Braneworld models belong to the most interesting cosmological models with extra dimensions. In this scenario, the observable universe is a (3+1)-dimensional hypersurface (three-brane) embedded in a higher-dimensional spacetime (bulk) [52,53] (for a recent review, see [54]). The stabilization mechanism of the extra dimensions in this scenario is an important issue. By introducing a bulk scalar field to the model, the classical stabilization mechanism for this scenario has been proposed [55] and intensively investigated (for example [56-58]). Since the boundary conditions of the bulk are also imposed, a quantum effect corresponding to the Casimir energy is generated and then used by letting it play an essential role in the stabilization mechanism [59-63]. It is important to note that there is a codimension-two brane in $6 \mathrm{D}$ gauge chiral supergravity model which proposes a stabilization mechanism for the extra dimensions from the brane-bulk couplings [49-51]. In this model, the extra dimension geometry is set to be a rug ball where the branes are located at the tips. This geometry allows one to construct the model in the presence of supersymmetries in the bulk, while supersymmetries break on the branes. The Casimir energy calculated from one-loop corrections of the bulk fields may also play the role of he cosmological constant to drive the late-time acceleration of the universe.

The classical stabilization mechanism in the braneworld scenarios is accomplished by the existence of the radion mass. However, the radion field shows the trend to have a negative mass square leading to tachyonic instabilities [64]. By including the Gauss-Bonnet term, the dynamics of the radion field will be affected such that, for positive GaussBonnet coupling, the radion mass increases and the interbrane distance becomes smaller as the coupling increases and the radion field can evolve to the region without tachyonic instabilities. For the negative coupling case, the radion field evolves to the tachyonic instability state $[65,66]$. Thus it turns out that the Gauss-Bonnet term with a positive coupling can help to stabilize the extra dimension.

In this paper, we will seek a way to restore the stabilization mechanism in the Casimir dark energy model by considering the modification of gravity instead of adding an exotic matter field. For the modified gravity, we will consider the generalization of Einstein gravity namely "Lovelock gravity" [67-69]. Lovelock gravity is a generalization of Einstein gravity in higher-dimensional spacetime which gives rise to Einstein gravity with a cosmological constant in fourdimensional spacetime. One of the important properties of this modified gravity is that it still provides the equation of motion with second order derivatives of the theory and satisfies the conservation equation of matter field; in other words, it satisfies the modified Bianchi identities. In fiveand six-dimensional spacetime, Lovelock gravity reduces to Einstein-Gauss-Bonnet (EGB) gravity which is Einstein gravity including a Gauss-Bonnet (GB) term. In fact, the GB term might also arise from string theory [70,71]. Therefore, it is worthwhile to investigate the effect of the GB term on the stabilization of the extra dimensions in the Casimir dark energy model and this is the aim of this work. We find the equations of motion in five-dimensional spacetime and then use numerical methods to show that the extra dimensions can be stabilized. For the numerical method, the minimum radion potential has to be positive and the potential must be deep enough to stabilize the extra dimension. This requires a fine tuning of the model parameters and the most sensitive one is the mass ratio parameter $\bar{\lambda}$. The other mechanisms which influence the stabilization mechanism are the spectrum of the particles to generate the Casimir energy as well as the initial value of the radion field.

The effective four-dimensional theory is obtained by Kaluza-Klein reduction [72-74]. By using this result, we show that the contribution from the GB term effectively slows down the radion field during the matter-dominated period so that it does not pass the point at which the minimum of the potential will arise before the minimum has formed. The field then is trapped at the minimum of the potential after the for- 
mation leading to the stabilization of the extra dimensions. We also investigate this mechanism in six-dimensional spacetime and show that the radius of the extra dimensions can be stabilized in the same manner as in the five-dimensional analysis.

The paper is organized as follows. We review the Casimir dark energy model in Sect. 2. The stabilization of the extra dimension when non-relativistic matter is not included is discussed and the destabilization mechanism of the extra dimension when the non-relativistic matter is taken into account is also discussed in this section. In Sect. 3, the Lovelock gravity theory in $(4+n)$-dimensional spacetime and EGB gravity are reviewed. The Kaluza-Klein reduction of EGB gravity theory is also reviewed in this section. In Sect. 4, we use the results of the two previous sections to modify the Casimir dark energy model by including the GB term and show how the GB term affects the dynamics of radion field in both fiveand six-dimensional spacetime. Finally, we conclude with the results in Sect. 6.

\section{Casimir dark energy model}

In this section, we will review one of the theoretical models, the so called "Casimir dark energy model" by following $[35,42]$. The stabilization mechanism of the extra dimension in this model is reviewed. The destabilization of the extra dimension when non-relativistic matter added is also reviewed.

\subsection{Casimir energy and its interpretation as dark energy}

The Casimir energy is the vacuum energy emerging from imposing boundary conditions on the quantum fluctuations of fields at small scales [75-78]. This energy is seen to be a physical energy since the Casimir force can be observed in terrestrial experiments $[79,80]$. In this subsection, we will review the mathematical calculation and physical description of the Casimir energy from the compactification of the extra dimension. Then we will review an interpretation of the Casimir energy as dark energy in order to drive the late-time acceleration of the universe. This dark energy model provides the mechanism for stabilizing the extra dimension. However, this mechanism will be destroyed when non-relativistic matter is added.

Generally, the Casimir energy can be derived in any number of extra dimensions. In this subsection we will consider an ansatz in which a single extra dimension is compactified as a circle, $S^{1}$, and five-dimensional spacetime can be thought of as the product space of the four-dimensional flat FLRW spacetime and this circle space. In six-dimensional spacetime, given by the product space of the four-dimensional flat
FLRW spacetime and a simple two-dimensional torus, $T^{2}$, the Casimir energy can easily be derived using a method analogous to the derivation in five-dimensional spacetime $[34,35]$. However, the calculation for a non-trivial twodimensional torus, for example a torus which is characterized by both its volume and its shape, will be more complicated since we need more complex mathematical tools [34,41]. In this paper we will use the results derived in five-dimensional spacetime to obtain the analogous results in six-dimensional spacetime with a simple torus, the torus which is characterized by only its volume. The line element of this ansatz can be written as

$d s^{2}=-d t^{2}+a^{2}(t) d \mathbf{x}^{2}+b(t)^{2} d y^{2}$,

where $a(t)$ denotes the scale factor of the 3-spatial noncompact directions and $b(t)$ denotes the scale factor of the compact fifth direction which is characterized the volume of the extra dimension. The range of the coordinate $y$ on the $S^{1}$ is $0 \leq y \leq 2 \pi$. Considering a simple massive scalar field living in this spacetime, the equation of motion for this scalar field is the Klein-Gordon equation,

$\left(\nabla_{A} \nabla^{A}-m^{2}\right) \phi=0$,

where $m$ is a mass and the uppercase Latin indices, $A, B$, $C, \ldots$ are five spacetime indices running on $\{0,1,2,3,5\}$. Since the fifth direction of the spacetime is compactified as a circle, we can impose the periodic boundary conditions of the scalar field as $\phi(y=0)=\phi(y=2 \pi)$. Since the Klein-Gordon equation has the form of a wave equation, the solution may be written as $\phi \propto e^{i\left(k_{\mu} x^{\mu}+k_{5} y\right)}$, where $k_{\mu}$ is the wave number in the non-compact directions and $k_{5}$ is the wave number in the compact direction. By imposing periodic boundary conditions, the wave number in the compact direction, $k_{5}$, becomes an integer and then the dispersion relation of the scalar field can be written as

$$
-k^{\mu} k_{\mu}=m^{2}+\frac{\tilde{n}^{2}}{b^{2}},
$$

where $\tilde{n} \in \mathbb{Z}$ is the momentum number in the compact direction. For standard quantum field theory in four-dimensional spacetime, the zero point energy, or vacuum energy, of a massive scalar field is

$\widehat{E}=\left(\frac{L}{2 \pi}\right)^{3} \int d^{3} k \frac{1}{2} \omega_{k}$,

where $\omega_{k}=\sqrt{k^{2}+m^{2}}$, from the dispersion relation, $-k^{\mu} k_{\mu}=m^{2}$, and $L^{3}$ is the spatial volume. We can use an analogous method to obtain the vacuum energy in the previous expression with $\omega_{k}=\sqrt{k^{2}+m^{2}+\tilde{n}^{2} / b^{2}}$, derived from the dispersion relation in Eq. (3). The integration of the compact direction becomes the summation over $\tilde{n}$. We assume that the relevant vacuum solution in the non-compact direction is the Cartesian product of flat 4-spacetime. This 
assumption is a basic assumption for calculating the Casimir energy in a higher-dimensional spacetime, as seen in the literature, for example $[34,82]$. Using this method the vacuum energy of the scalar field in our case can be written as

$\widehat{E}_{\text {Cas }}=\frac{1}{2}\left(\frac{L}{2 \pi}\right)^{3} \int d^{3} k \sum_{\tilde{n}} \sqrt{k^{2}+m^{2}+\frac{\tilde{n}^{2}}{b^{2}}}$.

The integration of the summation above seems to be divergent since $k$ runs from 0 to $\infty$. However, we can regularize this integration by using the Chowla-Selberg zeta function [81]. We will not show the explicit calculation for this regularization procedure. The detailed calculation can be found in [42]. The result of the regularization is finite and we now interpret this as the Casimir energy [34]. For the massless and massive scalar fields, the energy density of each component can be, respectively, written as

$$
\begin{aligned}
\rho_{\mathrm{Cas}}^{\text {massless }} & =\frac{\widehat{E}_{\mathrm{cas}}}{L^{3} 2 \pi b}=\frac{2 \Gamma(5 / 2) \zeta(5)}{\Gamma(-1 / 2)(2 \pi b)^{5} \pi^{2}}, \\
\rho_{\mathrm{Cas}}^{\text {massive }} & =\frac{-2(m b)^{5 / 2}}{(2 \pi b)^{5}} \sum_{\tilde{n}=1}^{\infty} \tilde{n}^{-5 / 2} K_{5 / 2}(2 \pi b m \tilde{n}),
\end{aligned}
$$

where $\zeta$ is the zeta function, $\Gamma$ is the gamma function, and $K_{v}(x)$ is a modified Bessel function. The main results of these energy densities is that they are proportional to $1 / b^{5}$. This is one of characteristic behaviors of the Casimir energy, with $\rho_{\text {Cas }} \propto 1 / a^{D}$, where $a$ is the distance between the boundaries and $D$ in a number of all spacetime dimensions. It is not surprising, since the procedure used to calculate the Casimir energy is the same as the one for calculating in the case of parallel conducting plates. It is important to note that, by relaxing our assumption of the vacuum solution in a non-compact direction, one can consider the vacuum energy in FLRW spacetime. It was found that the physical vacuum energy can be obtained by subtracting the bare vacuum energy of Minkowski spacetime from the FLRW and then renormalizing by adjusting non-covariant counterterms $[83,84]$. As a result, the physical vacuum energy density is proportional to the Hubble parameter square $\rho_{v a c} \propto H^{2}$. For a more general consideration, the covariant approach to calculate the renormalized energy-momentum tensor of vacuum was investigated [85]. As a result, $\left\langle T_{\mu \nu}\right\rangle_{v a c} \propto G_{\mu \nu}$ where $G_{\mu \nu}$ is component of Einstein tensor. It is obvious that this physical vacuum energy is totally different from the Casimir energy and must be distinguished from the Casimir energy. Since this physical energy influences the dynamics of the universe, it may yield the stabilization mechanism of the extra dimensions. We leave this investigation for further work since our aim is to investigate the effect of the GaussBonnet terms on the stabilization mechanism of the extra dimensions.
The contribution of the graviton to the Casimir energy in five-dimensional spacetime is $\rho_{\text {graviton }}=5 \rho_{\text {scalar }}$ [82] where the number 5 comes from the number of degrees of freedom of the graviton in five-dimensional spacetime. This result can be generalized to other bosonic fields using knowledge of supersymmetry multiplets. It is found that the contribution from other bosonic fields to the Casimir energy can be written in terms of the scalar contribution as $\rho_{\text {boson }}=N_{b} \rho_{\text {scalar }}$, where $N_{b}$ is the number of degrees of freedom of the bosonic fields. This generalization can be applied to fermionic fields and it is found that the contribution from the fermionic fields to the Casimir energy is given by an equivalent expression, but with a negative sign, $\rho_{\text {fermion }}=-N_{f} \rho_{\text {scalar }}$ [34], where $N_{f}$ is a number of degrees of freedom of the fermionic fields. In order to interpret the Casimir energy as a cosmological constant, the total Casimir energy density can be expected to occur as the potential term of the radion field in four-dimensional spacetime. The radion field with a potential contributing from the Casimir energy density can play the role of dark energy if there exists a positive minimum of the potential. In order to obtain the positive minimum of the potential, one has to choose the proper contribution from both massive/massless bosons and fermions as well as the mass ratio between them $\bar{\lambda}=m_{b} / m_{f}$. Since the metric $g_{A B}$ is the dynamical field of our ansatz, the massless bosonic contribution must come from, at least, the graviton. As we have mentioned above, the number of degrees of freedom of the graviton is $N_{b}=5$. For massless fermionic fields, we assume that there are two quantum fluctuations of the Dirac fermionic fields living in the bulk. In five-dimensional spacetime, the physical degree of freedom of the Dirac fermions is four. Therefore, the total number of degrees of freedom for massless fermionic field is $N_{f}=8$. For massive fermions, with mass $m_{f}$, we also use the same assumption as for the massless ones. Therefore, the total number of degrees of freedom of the massive fermionic field is $\tilde{N}_{f}=8$. The massive boson contribution may come from eight massive scalar fields with mass $m_{b}$. By using this particular choice, the total Casimir energy density can be written as

$$
\begin{aligned}
\rho_{\text {Cas }} & =\rho_{\text {boson }}^{\text {massless }}+\rho_{\text {fermion }}^{\text {massless }}+\rho_{\mathrm{boson}}^{\text {massive }}+\rho_{\text {fermion }}^{\text {massive }}, \\
& =-3 \rho_{\mathrm{Cas}}^{\text {massless }}+8 \rho_{\mathrm{Cas}}^{\text {massive }}\left(m_{b}\right)-8 \rho_{\mathrm{Cas}}^{\text {massive }}\left(m_{f}\right) .
\end{aligned}
$$

It is important to note that there is no unique choice of bulk particle spectrum for this purpose; other combinations of the fields can probably also create a minimum for the vacuum energy [35]. Note also that we do not attempt to justify the existence of these bulk fields phenomenologically because we seek only to demonstrate that a stabilization mechanism of the extra dimensions can be achieved if these fields are present. 
Fig. 1 The evolution of the radius of the extra dimension (in the left panel) and the scale factor (in the right panel) in the Casimir dark energy model without non-relativistic matter. From the left panel, we see that the radius of the extra dimension can be stabilized and from the right panel we see that our 3 -spatial universe is accelerated

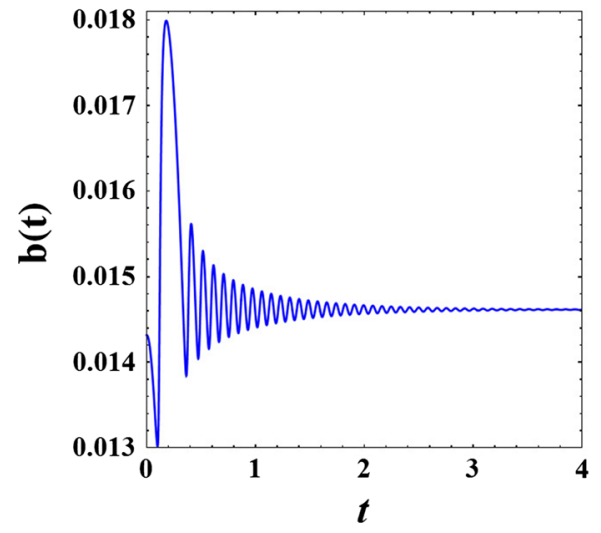

\subsection{Dynamics of Casimir dark energy}

In order to obtain the dynamics of the Casimir dark energy, we add the energy-momentum tensor contributing from the Casimir effect into the Einstein field equation. The general form of the Casimir energy-momentum tensor which is compatible with the metric in Eq. (1) can be written as [35]

$T_{\nu(\text { Cas })}^{\mu}=\operatorname{diag}\left(-\rho_{\text {Cas }}, p_{a}, p_{a}, p_{a}, p_{b}, \ldots, p_{b}\right)$

where $p_{a}$ and $p_{b}$ are the Casimir pressure in the non-compact and compact dimensions, respectively. These pressures can be defined as [35]

$p_{a} \equiv-\frac{\partial}{\partial V_{a}}\left(\rho_{\text {Cas }} V_{a}\right)$,

$p_{b} \equiv-\frac{\partial}{\partial V_{b}}\left(\rho_{\text {Cas }} V_{b}\right)$,

where $V_{a} \propto a^{d-n}$ and $V_{b} \propto b^{n}$. Here, $d$ is the number of spatial dimensions, $n$ is the number of the extra dimensions and $d=4, n=1$ for this model. It is important to note that the equation of state (eos) defined in Eqs. (10) and (11) can be applied to any perfect fluid which obeys the equation $p=w \rho$ where $p$ is the pressure, $\rho$ is the energy density and $w$ is the equation of state parameter. For example, one can check this definition by substituting $\rho=\rho_{0} a^{-(1+w) / 3}$ into Eq. (10), and then one can obtain $p=w \rho$.

Since the Casimir energy density, $\rho_{\text {Cas }}$, depends only on $b$, the pressure in the non-compact and compact directions can, respectively, be written as $p_{a}=-\rho_{\text {Cas }}$ and $p_{b}=-\rho_{\text {Cas }}-$ $b \partial_{b} \rho_{\text {Cas }}$. The equation of state for the cosmological constant is $p_{\Lambda}=-\rho_{\Lambda}$. It is found that the equation of state of the Casimir energy has the same form as the equation of state of the cosmological constant. It is convenient to consider the general form of the metric with $n$ extra dimensions as

$d s^{2}=-d t^{2}+a^{2}(t) d \mathbf{x}^{2}+b(t)^{2} d \mathbf{y}^{2}$,

where $\mathbf{y}$ denotes the coordinates in the $n$-extra dimensions. By using this metric, the conservation equation of the energy momentum tensor, $\nabla_{\mu} T_{\nu}^{\mu}$, reads

$\dot{\rho}_{\text {Cas }}+3 H_{a}\left(\rho_{\text {Cas }}+p_{a}\right)+n H_{b}\left(\rho_{\text {Cas }}+p_{b}\right)=0$,

where $H_{a}=\dot{a} / a$ and $H_{b}=\dot{b} / b$. Using the metric in Eq. (12) and the energy-momentum tensor in Eq. (9), the fields equation [86] are

$$
\begin{gathered}
3 H_{a}^{2}+\frac{n}{2}(n-1) H_{b}^{2}+3 n H_{a} H_{b}=M_{*}^{-(n+2)} \rho_{\mathrm{Cas}} \\
n \frac{\ddot{b}}{b}+2 \frac{\ddot{a}}{a}+\frac{n}{2}(n-1) H_{b}^{2}+H_{a}^{2}+2 n H_{a} H_{b}=-M_{*}^{-(n+2)} p_{a} \\
(n-1)\left(\frac{\ddot{b}}{b}+\frac{(3 n-2)}{2} H_{b}^{2}+3 H_{a} H_{b}\right) \\
+3 \frac{\ddot{a}}{a}+3 H_{a}^{2}=-M_{*}^{-(n+2)} p_{b}
\end{gathered}
$$

where $M_{*}$ is the mass scale in (4+n)-dimensional spacetime. Note that we generalized the Einstein field equations into $(4+n)$-dimensional spacetime for convenience.

For $n=1$, the numerical results of these equations are shown in Fig. 1. From this figure, we can see that the radius of the extra dimension can be stabilized at $b(t) \sim 0.0145$ and the scale factor is accelerated. In order to get the positive minimum in which the radion can be stabilized, we must choose the value of $\bar{\lambda}$ from a very narrow range, $0.516 \leq \bar{\lambda} \leq$ 0.527 . In these numerical results, we have chosen $\bar{\lambda}=0.516$, which is the lower bound of the range. It is important to note that a fine tuning problem is also found in this model since one needs to fine tune the parameter $\bar{\lambda}$ for three digits to stabilize the extra dimension. This fine tuning problem is different from the one from the cosmological constant model since the parameter is fine tuned to stabilize the extra dimension, while the initial value of the cosmological constant is fine tuned to obtain the consistent value at the present time. For this model, the value dark energy at the present time gives a prediction of the mass of the fermionic bulk field [35]. To obtain the realistic cosmological history of the universe, the contribution from non-relativistic matter must be added. The 
Fig. 2 The evolution of the radius of the extra dimension (in the left panel) and the scale factor (in the right panel) in the Casimir dark energy model including non-relativistic matter. From the left panel, we see that the radius of the extra dimension cannot be stabilized and from the right panel we see that our 3-spatial universe cannot be accelerated

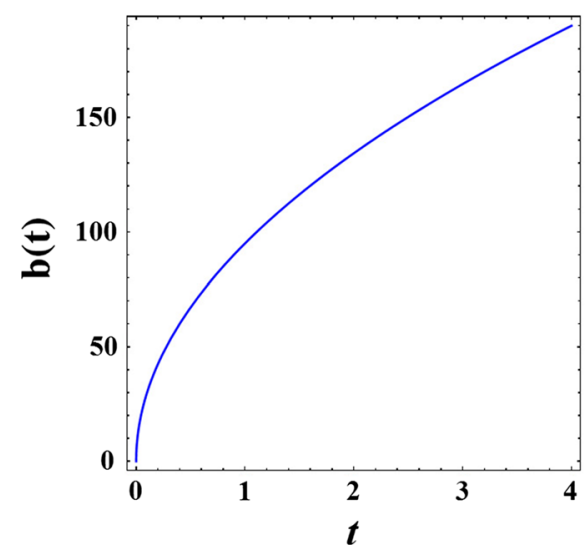

Einstein equations including non-relativistic matter in fivedimensional spacetime are

$3 H_{a}^{2}+3 H_{a} H_{b}=M_{*}^{-3}\left(\rho_{\text {Cas }}+\rho_{m}\right)$,

$\frac{\ddot{b}}{b}+2 \frac{\ddot{a}}{a}+H_{a}^{2}+2 H_{a} H_{b}=-M_{*}^{-3} p_{a}$,

$3 \frac{\ddot{a}}{a}+3 H_{a}^{2}=-M_{*}^{-3} p_{b}$,

where $\rho_{m}$ is the energy density of non-relativistic matter. The energy density of non-relativistic matter in $(4+n)-$ dimensional spacetime, obeying the conservation equation $\dot{\rho}_{m}+3 H_{a}+n H_{b}=0$, can be written as

$\rho_{m}=\left(\frac{b_{\min }}{b}\right)^{n} \frac{\rho_{m} 0}{a^{3}}$,

where $\rho_{m 0}$ is the energy density of non-relativistic matter at the present epoch (corresponding to $b=b_{\min }$ and $a=1)$. From the observational data [3], $\rho_{m 0}^{(4)}=$ $(2.8 / 7.2) \rho_{\Lambda}=(2.8 / 7.2)\left(2.3 \times 10^{-3} \mathrm{eV}\right)^{4}$. Therefore $\rho_{m 0}=$ $(2.8 / 7.2) \rho_{\text {Cas }}\left(b=b_{\text {min }}\right)$, since $\rho_{\Lambda}=\left(2 \pi b_{\text {min }}\right)^{n} \rho_{\text {Cas }}(b=$ $\left.b_{\text {min }}\right)$ and $\rho_{m 0}^{(4)}=\left(2 \pi b_{\min }\right)^{n} \rho_{m 0}$. Using this relation the energy density of non-relativistic matter in $(4+n)$ dimensional spacetime can be written as

$\rho_{m}=\frac{2.8}{7.2}\left(\frac{b_{\min }}{b}\right)^{n} \rho_{\text {Cas }}\left(b=b_{\min }\right) a^{-3}$.

The numerical results of the evolution of $b(t)$ and $a(t)$ for Eqs. (17)-(19) are shown in Fig. 2. We can see that the radius of the extra dimension cannot be stabilized and the scale factor will not be accelerated. The destabilization of the extra dimension will be examined by considering the potential of the radion field in four-dimensional spacetime. The minimum of the potential for the radion field does not exist at early times since the contribution of non-relativistic matter is dominant. Therefore, the radion field will roll down and pass away from the minimum point before it exits [35]. We will consider this issue in detail in Sect. 4. By including the effect of an aether field, the stabilization of the extra dimension can be restored [42]. However, the aether field itself is not stable [45-48]. Hence the stabilization mechanism obtained by including the aether field may not be worthy of the effort.

\section{Einstein-Gauss-Bonnet theory}

In this section, we briefly review the concept of Lovelock invariance by following [67-69]. This leads to a generalization of Einstein gravity keeping second order equations of motion and covariant conservation of the matter field. This generalization does not change Einstein gravity in fourdimensional spacetime but gives a non-trivial modification when the theory is considered in higher-dimensional spacetime. For five- or six-dimensional spacetime, it is well known that this generalization is Einstein-Gauss-Bonnet (EGB) theory. We will review this theory especially in this section since we restrict our attention to a Casimir dark energy model emerging from a compactification of spacetime dimensions from five and six to four. The Kaluza-Klein compactification of EGB theory is also reviewed in the final part of this section.

We begin this section with considering the EGB action in D-dimensional spacetime as follows:

$S_{E G B}=\int d^{D} x \sqrt{-g}\left(\frac{M_{*}^{D-2}}{2}\left(-2 \Lambda+R+\lambda^{2} \mathcal{G}\right)+L_{m}\right)$,

where $M_{*}$ is the fundamental mass scale of the D-dimensional theory, $\Lambda$ is the cosmological constant corresponding to the zero order Lovelock Lagrangian, and $R$ is the Ricci scalar corresponding to the first order Lovelock Lagrangian, $\mathcal{G}$ is the Gauss-Bonnet term corresponding to the second order Lovelock Lagrangian; $\lambda$ is a coupling constant parameterizing the presence of the Gauss-Bonnet term. Note that $L_{m}$ is the Lagrangian of non-relativistic matter. The Gauss-Bonnet 
term can be defined as

$\mathcal{G}=R^{2}-4 R^{\nu_{1} \nu_{2}} R_{\nu_{1} \nu_{2}}+R^{\mu_{1} \mu_{2}} \mu_{3} \mu_{4} R^{\mu_{3} \mu_{4}} \mu_{1} \mu_{2}$.

By varying the action in Eq. (22) with respect to the metric, the generalized version of the Einstein equation can be written as

$\Lambda \delta_{v}^{\mu}+G_{v}^{\mu}+\lambda^{2} H_{v}^{\mu}=M_{*}^{2-D} T_{v}^{\mu(m)}$,

where $T_{\mu \nu}^{(m)}=2 \delta\left(\sqrt{-g} L_{m}\right) / \delta g^{\mu \nu}$ is the energy-momentum tensor of the matter field. $H_{v}^{\mu}$ is the second order Lanczos tensor obtained by varying the Gauss-Bonnet term with respect to the metric. This tensor can be written as

$$
\begin{aligned}
H_{v}^{\mu}= & 2\left(R^{\mu \mu_{1} \mu_{2} \mu_{3}} R_{v \mu_{1} \mu_{2} \mu_{3}}-2 R^{\mu_{1} \mu_{2}} R_{\mu_{1} v \mu_{2}}^{\mu}\right. \\
& \left.-2 R^{\mu \mu_{1}} R_{v \mu_{1}}+R R_{v}^{\mu}\right)-\frac{1}{2} \mathcal{G} \delta_{v}^{\mu} .
\end{aligned}
$$

It is important to note that Lovelock invariance can be considered in terms of the vierbein or tetrad formalism [8789]. In this formalism, the Lovelock Lagrangian can be constructed from powers of the curvature two-form. The advantages of this formalism are that it provides a clear geometric interpretation and it is easy to show that the equations of motion corresponding to the Lovelock Lagrangian are of second order.

Since observations suggest that the universe is fourdimensional, any extra dimensions have to be compactified. In this subsection, we consider the Kaluza-Klein compactification from (4+n)-dimensional spacetime to fourdimensional spacetime where $n$ is the number of the extra dimensions $[72,73,73]$. In this paper, we will review a special case of the generalized Kaluza-Klein compactification [89]. We also restrict our attention only to the diagonal metric of the internal extra dimensions for simplicity. Note that the existence of off-diagonal parts of the metric in fivedimensional spacetime corresponds to the existence of a gauge field, $A_{\mu}$, in four-dimensional effective theory. Since the goal of this paper is to investigate the stabilization mechanism of the extra dimensions, more degrees of freedom in four-dimensional theory may lead to difficulties in analyzing the result. This investigation is outside the scope of this work. It is important to note that one can eliminate the gauge field from our consideration by requiring more symmetry, for example, $Z_{2}$ symmetry of the interval $d s^{2}$. Since this symmetry does not affect the form of the Casimir energy density [34], the interpretation of the cosmological constant in terms of the Casimir energy is still applicable. The metric for this ansatz can be written as

$$
\begin{aligned}
d s^{2} & =g_{A B} d x^{A} d x^{B}, \\
& =e^{-\sqrt{\frac{n}{n+2}} \phi} \bar{g}_{\mu \nu} d x^{\mu} d x^{\nu}+e^{\sqrt{\frac{4}{n(n+2)}} \phi} \tilde{g}_{a b} d y^{a} d y^{b},
\end{aligned}
$$

where the indices $A, B, \ldots$ run over the whole D-dimensional spacetime, the indices $\mu, v, \ldots$ run over the $(3+1)$ dimensional spacetime and the indices $a, b, \ldots$ run over the internal space in $n$ dimensions. For simplicity, we assume that $\bar{g}_{\mu \nu}$ is diagonal and $\phi$ depends only on the external spacetime coordinates, $\phi=\phi\left(x^{\mu}\right)$. The conformal factor, $e^{-\sqrt{\frac{n}{n+2}} \phi}$, of the metric $\bar{g}_{\mu \nu}$ is inserted for convenience in the calculation procedure. Note that the metric $\tilde{g}_{a b}$ is assumed to be a flat Euclidean metric which is the one we have used for calculating the Casimir energy density. More detailed calculations with generic Euclidean metrics are given in the appendix. Using this ansatz together with the calculation in the standard procedure of the Kaluza-Klein reduction, the EGB action with matter in D-dimensional spacetime in Eq. (22) can be reduced to

$$
\begin{aligned}
S_{E G B}= & \int d^{4} x \sqrt{-\bar{g}} \frac{M_{P l}^{2}}{2}\left[\lambda ^ { 2 } e ^ { \sqrt { \frac { n } { ( n + 2 ) } } \phi } \left(\overline{\mathcal{G}}-4 f_{1} \bar{G}^{\mu \nu} \partial_{\mu} \phi \partial_{\nu} \phi\right.\right. \\
& \left.-2 f_{2}(\partial \phi)^{2} \square \phi-f_{3}(\partial \phi)^{2}(\partial \phi)^{2}\right)-2 \Lambda e^{-\sqrt{\frac{n}{(n+2)}} \phi} \\
& \left.+\left(\bar{R}-\frac{1}{2}(\partial \phi)^{2}\right)+\frac{2(2 \pi)^{n}}{M_{P l}^{2}} e^{-\sqrt{\frac{n}{(n+2)}} \phi} L_{m}\right], \quad(27)
\end{aligned}
$$

where $f_{1}, f_{2}$, and $f_{3}$ are functions depending on $n$, defined in the appendix. The EGB theory in five-dimensional spacetime also reduces to

$$
\begin{aligned}
S_{E G B}= & \int d^{4} x \sqrt{-\bar{g}}\left[\frac{M_{P l}^{2}}{2}\left\{\bar{R}-2 \Lambda e^{-\frac{\psi}{\psi_{0}}}+\lambda^{2} e^{\frac{\psi}{\psi_{0}}} \overline{\mathcal{G}}\right\}\right. \\
& +\lambda^{2} e^{\frac{\psi}{\psi_{0}}}\left(\frac{4}{3} \bar{G}^{\mu \nu} \partial_{\mu} \psi \partial_{\nu} \psi+(\partial \psi)^{2} \frac{\square \psi}{\psi_{0}}\right) \\
& \left.-\frac{1}{2}(\partial \psi)^{2}+2 \pi e^{-\frac{\psi}{\psi_{0}}} L_{m}\right],
\end{aligned}
$$

where we have rescaled the scalar field as follows:

$\phi=\frac{\sqrt{2} \psi}{M_{P l}}=\sqrt{3} \frac{\psi}{\psi_{0}}$.

In order to relate the radius of the extra dimension $b(t)$ with the scalar $\phi(t)$, we note that the explicit relation can be expressed as

$b(t)=e^{\frac{\phi}{\sqrt{n(n+2)}}}$.

\section{Casimir dark energy model with Einstein-Gauss-Bonnet theory}

In this section, we will consider the EGB theory with the action (22). In our ansatz, the cosmological constant is interpreted as the Casimir energy density as follows: $M_{*}^{3} \Lambda=$ $\rho_{\text {Cas }}=L_{\text {Cas }}(\phi)$. By substituting this result into Eq. (22), the 
Fig. 3 The evolution of the radius of the extra dimension (in the left panel) and the scale factor (in the right panel) in the Casimir dark energy model including non-relativistic matter and the Gauss-Bonnet term. From the left panel, we see that the radius of the extra dimension can be stabilized and from the right panel we see that our 3 -spatial universe can be accelerated. We have used $\lambda=0.1$ in this simulation

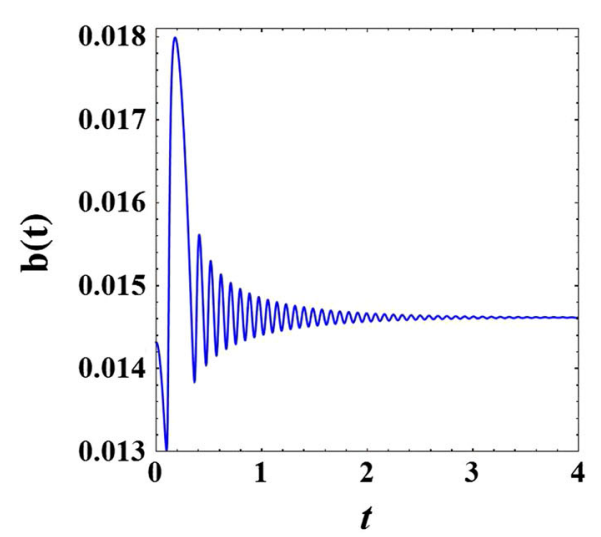

action can be expressed as

$S_{5}=\int d^{5} x \sqrt{-g}\left(\frac{M_{*}^{3}}{2}\left(R+\lambda^{2} \mathcal{G}\right)-L_{\text {Cas }}(\phi)+L_{m}\right)$.

The equations of motion are obtained from Eq. (24) as

$G_{v}^{\mu}+\lambda^{2} H_{v}^{\mu}=M_{*}^{-3}\left(T_{v(\text { Cas })}^{\mu}+T_{v(m)}^{\mu}\right)$

where $T_{v(\text { Cas })}^{\mu}$ is defined in Eq. (9). By using the metric in Eq. (12), each component of the above equation can be expressed as

$3 H_{a}^{2}+3 H_{a} H_{b}+12 \lambda^{2} H_{a}^{3} H_{b}=M_{*}^{-3}\left(\rho_{\mathrm{Cas}}+\rho_{m}\right)$,

$\frac{\ddot{b}}{b}+2 \frac{\ddot{a}}{a}+H_{a}^{2}+2 H_{a} H_{b}+\lambda^{2}\left(8 H_{a} H_{b} \frac{\ddot{a}}{a}+4 H_{a}^{2} \frac{\ddot{b}}{b}\right)$

$=-M_{*}^{-3} p_{a}$,

$3 \frac{\ddot{a}}{a}+3 H_{a}^{2}+\lambda^{2} H_{a}^{2} \frac{\ddot{a}}{a}=-M_{*}^{-3} p_{b}$.

The numerical results of these equations of motion are illustrated in Fig. 3. We can see that the extra dimension can be stabilized and the universe is accelerated. Note that we use $\lambda=0.1$ in the simulation. The range in which the extra dimension can be stabilized is approximately $0.005 \leq \lambda^{2} \leq$ 5.0. This range depends on the initial velocity of the extra dimension, $\dot{b}_{i}$. By setting $\lambda=1$, the initial velocity required is $\dot{b}_{i}<0.002$ in order to stabilize the extra dimension. To compare the results of the model with the standard history of the universe, we need to include the contribution of radiation. This contribution may alter the behavior of the stabilization mechanism. This modification can be done by changing the initial conditions to their values at the radiation-domination epoch and substituting the energy density and pressure of the radiation instead of matter. By doing this, we found that the stabilization mechanism still hold. In order to see how the stabilization mechanism can be restored, we will analyze this mechanism using the equations of motion in fourdimensional spacetime.

\section{Dynamics in radion picture}

From Eq. (31), the cosmological constant term can be interpreted as a Casimir energy density $L_{\text {Cas }}^{(5)}(\phi)$. The effect of dimensional reduction to this term will come only from the part of $\sqrt{-g}$. In fact, the amount of the factor is the same as for the matter term as $2 \pi e^{-\frac{\psi}{\psi_{0}}}$. By using the rescaling field in Eq. (29), the reduced action of this term becomes $2 \pi e^{-\frac{\psi}{\psi_{0}}} L_{\text {Cas }}^{(5)}(\psi)$. Since this term does not contain the contribution of the derivative of the scalar field, we can promote it to the potential term of the scalar field as $V(\psi)=$ $2 \pi e^{-\frac{\psi}{\psi_{0}}} L_{\text {Cas }}^{(5)}(\psi)=e^{-2 \frac{\psi}{\psi_{0}}} L_{\text {Cas }}^{(4)}(\psi)$, where we have used the relation of the energy densities in five-dimensional and four-dimensional spacetime, $\rho^{(4)}=2 \pi b \rho^{(5)}$. By using the reduced action in Eq. (28) and our interpretation of the cosmological constant term above, the reduced action in fourdimensional spacetime can be written as

$$
\begin{aligned}
S^{(4)}= & \int d^{4} x \sqrt{-\bar{g}}\left[\frac{M_{P l}^{2}}{2}\left\{\bar{R}+\lambda^{2} e^{\frac{\psi}{\psi_{0}}} \overline{\mathcal{G}}\right\}-\frac{1}{2}(\partial \psi)^{2}-V(\psi)\right. \\
& \left.+\frac{L_{m}^{(4)}}{e^{2 \frac{\psi}{\psi_{0}}}}+\lambda^{2} e^{\frac{\psi}{\psi_{0}}}\left(\frac{4}{3} \bar{G}^{\mu \nu} \partial_{\mu} \psi \partial_{\nu} \psi+(\partial \psi)^{2} \frac{\square \psi}{\psi_{0}}\right)\right] .
\end{aligned}
$$

Note that, without the last two terms and the potential, this action can be derived from the heterotic or type IIB string and there is a tachyonic instability for the tensor mode and a tachyon free case for the scalar mode [90,91]. This model has also been investigated in Bianchi type I [92] and type IX [93]. It is found that this kind of the model seems to be unstable. However, by including the last two terms, the stability conditions in the tensor mode will be modified. Without the potential term, the theory appears to be stable but the 
matter phase is absent $[94,95]$. Adding the potential term corresponding to the Casimir energy may therefore provide the required matter phase in the history of the universe. We leave this investigation for further work. The components of the equation of motion obtained by varying this action with respect to $g_{\mu \nu}$ can be written as

$$
\begin{aligned}
& 3 M_{P l}^{2} H_{a}^{2}=\rho_{\psi}+e^{-2 \frac{\psi}{\psi_{0}}} \rho_{m}^{(4)}, \\
& M_{P l}^{2}\left(3 H_{a}^{2}+2 \dot{H}_{a}\right)=-p_{\psi},
\end{aligned}
$$

where

$$
\begin{aligned}
\rho_{\psi}= & \frac{1}{2} \dot{\psi}^{2}+V \\
& +\tilde{\lambda}^{2} e^{\frac{\psi}{\psi_{0}}}\left(-8 H_{a}^{3} \frac{\dot{\psi}}{\psi_{0}}+12 H_{a}^{2} \frac{\dot{\psi}^{2}}{\psi_{0}^{2}}+6 H_{a} \frac{\dot{\psi}^{3}}{\psi_{0}^{3}}-\frac{\dot{\psi}^{4}}{\psi_{0}^{4}}\right), \\
p_{\psi}= & \frac{1}{2} \dot{\psi}^{2}-V \\
& +\tilde{\lambda}^{2} e^{\frac{\psi}{\psi_{0}}}\left(\frac{16}{3} H_{a}\left(H_{a}^{2}+\dot{H}_{a}\right) \frac{\dot{\psi}}{\psi_{0}}-\frac{4}{3}\left(H_{a}^{2}+2 \dot{H}_{a}\right) \frac{\dot{\psi}^{2}}{\psi_{0}^{2}}\right. \\
& \left.-\frac{8}{3} H_{a} \frac{\dot{\psi}^{3}}{\psi_{0}^{3}}-\frac{\dot{\psi}^{4}}{\psi_{0}^{4}}+\frac{2}{3}\left(4 H_{a}^{2}-8 H_{a} \frac{\dot{\psi}}{\psi_{0}}-\frac{3 \dot{\psi}^{2}}{\psi_{0}^{2}}\right) \frac{\ddot{\psi}}{\psi_{0}}\right) .
\end{aligned}
$$

The conservation of the energy-momentum tensor provides the field equation for the radion field as

$\ddot{\psi}+3 H_{a} \dot{\psi}=-V_{\text {eff }}^{\prime}+2 e^{-2 \frac{\psi}{\psi_{0}}} \frac{\rho_{m}^{(4)}}{\psi_{0}}$,

where

$$
\begin{aligned}
V_{\mathrm{eff}}^{\prime}= & V^{\prime}+\tilde{\lambda}^{2} e^{\frac{\psi}{\psi_{0}}}\left(-\frac{8}{\psi_{0}} H_{a}^{2}\left(H_{a}^{2}+\dot{H}_{a}\right)\right. \\
& +8 H_{a}\left(3 H_{a}^{2}+2 \dot{H}_{a}\right) \frac{\dot{\psi}}{\psi_{0}^{2}}+\left(22 H_{a}^{2}+6 \dot{H}_{a}\right) \frac{\dot{\psi}^{2}}{\psi_{0}^{3}} \\
& \left.-\frac{\dot{\psi}^{4}}{\psi_{0}^{5}}+4\left(2 H_{a}^{2}+3 H_{a} \frac{\dot{\psi}}{\psi_{0}}-\frac{\dot{\psi}^{2}}{\psi_{0}^{2}}\right) \frac{\ddot{\psi}}{\psi_{0}^{2}}\right), \\
\tilde{\lambda}^{2}= & \psi_{0}^{2} \lambda^{2}=\frac{3}{2} M_{P l}^{2} \lambda^{2} .
\end{aligned}
$$

Considering Eqs. (37) and (39) with $\tilde{\lambda}=0$, neglecting the effect of the Gauss-Bonnet term, one can see that the contribution from the matter field will dominate at early times since $\rho_{m}^{(4)} \propto a^{-3}$ and $a \ll 1$. Therefore, the minimum of the potential will disappear. From Eqs. (41) and (42) with $\tilde{\lambda}=0$, the contribution from the matter field also increases the slope of the potential. Hence, the radion field will rapidly roll down and then pass away from the point at which the minimum of the potential will arise before the minimum has formed. This is the destabilization mechanism of the extra dimension discussed in [35].
Now we will see how the Gauss-Bonnet contribution alters the dynamics of the radion field. From Eq. (37), the effect of the Gauss-Bonnet term does not significantly change the existence of the potential minimum at early times since the effect of the matter field is still dominant. However, the contribution of the Gauss-Bonnet term can significantly change the dynamics of the radion field through the slope of the potential as seen in Eq. (42). Initially, the radion field begins at some points of the potential away from the minimum with a tiny fraction velocity, $\dot{\psi} / \psi_{i} \ll 0$. Note that this assumption is also required in order to stabilize the extra dimension in normal Casimir dark energy models. Therefore, the first term from the Gauss-Bonnet contribution in Eq. (42) is dominant and effectively reduces the slope of the potential corresponding to a reduction of the magnitude of the force acting on the radion field. Note that, initially, $\ddot{a} / a=\dot{H}_{a}+H_{a}^{2}<0$ and $V^{\prime}<0$. This term effectively slows down the radion field and then the radion field does not pass the point at which the minimum of the potential will arise before the minimum has formed.

The value of the potential minimum is very important to address the cosmological constant problem. As we have mentioned in Sect. 2, the existence of a positive minimum of the potential depends on the spectrum of particles contributing to the Casimir energy. By fixing the particle spectrum, the value of the potential minimum can be adjusted by the mass ratio $\bar{\lambda}=m_{b} / m_{f}$. The value of $\bar{\lambda}$ may be obtained from supersymmetric theories, but in this work, we do not try to address this issue. We choose $\bar{\lambda}$ as the lower bound $(\bar{\lambda}=0.516)$ of the range in which the potential minimum can exist. By using the approximation, $b m_{f}<<1$, it is found that $V_{\min } \propto m_{f}^{D}$ and $b_{\min }=e^{\psi / \psi_{0}} \propto m_{f}^{-1}[35,41]$. In order to find the constant of proportionality, one can use the numerical method of the dynamical equations. Therefore, one can determine the fermion mass $m_{f}$ and the radius of the extra dimension $b_{\min }$ from the observed value of the cosmological constant. It turns out that $m_{f} \sim 4.18 \times 10^{-2} \mathrm{eV}$ and $b_{\min } \sim 2.75 \times 10^{-6} \mathrm{~m}$ [42]. Note that the contribution from the Gauss-Bonnet term does not alter this calculation since it vanishes when the radion stabilizes at the minimum of the potential. One may interpret this kind of fermion as the sterile neutrino, which may play the role of dark matter [35]. By using this value of $b_{\min }$ together with the relation $M_{P l}^{2}=M_{*}^{2+n}\left(2 \pi b_{\min }\right)^{n}$, the quantum gravity scale in the bulk is $M_{*} \sim 1.19 \times 10^{9} \mathrm{GeV}$. This mass scale does not provide a solution for the mass hierarchy problem. In order to address this problem, one may consider a theory in which the number of spacetime dimensions is larger than five. As discussed in Sect. 3, the contribution from the GaussBonnet term can be applied for the five- or six-dimensional cases. Therefore, it is worthwhile to investigate whether the stabilization mechanism still works in six-dimensional spacetime. 
In six-dimensional spacetime, the calculation can be evaluated in the same manner as in the five-dimensional case. We show here only the reduced action and the significant changes of the equations of motion. The reduced action from six-dimensional spacetime can be written as

$$
\begin{aligned}
S^{(4)}= & \int d^{4} x \sqrt{-\bar{g}}\left[\frac{M_{P l}^{2}}{2}\left\{\bar{R}+\lambda^{2} e^{\frac{\psi}{\psi_{0}}} \overline{\mathcal{G}}\right\}-\frac{1}{2}(\partial \psi)^{2}-V(\psi)\right. \\
& \left.+\lambda^{2} e^{\frac{\psi}{\psi_{0}}}\left(\bar{G}^{\mu \nu} \partial_{\mu} \psi \partial_{\nu} \psi-\frac{(\partial \psi)^{2}(\partial \psi)^{2}}{4 \psi_{0}^{2}}\right)+\frac{L_{m}^{(4)}}{e^{2 \frac{\psi}{\psi_{0}}}}\right],
\end{aligned}
$$

where $V(\psi)=(2 \pi)^{2} e^{-\frac{\psi}{\psi_{0}}} L_{\text {Cas }}^{(6)}(\psi)=e^{-2 \frac{\psi}{\psi_{0}}} L_{\text {Cas }}^{(4)}(\psi)$ and the constant $\psi_{0}$ is now redefined as $\psi_{0}=M_{P l}$. The fields $\phi$ and $\psi$ are related via $\phi=\sqrt{2} \psi / \psi_{0}$. Comparing this to the reduced action from five-dimensional spacetime in Eq. (36), the higher-derivative part coming from the Gauss-Bonnet term differs; it is proportional to $(\partial \psi)^{2}(\partial \psi)^{2}$ for the sixdimensional case and it is proportional to $(\partial \psi)^{2} \square \psi$ for fivedimensional case. Using the same procedure for finding the equations of motion in the five-dimensional case, the energy density, pressure, and $V_{\text {eff }}^{\prime}$ can be rewritten as

$$
\begin{aligned}
\rho_{\psi}= & \frac{1}{2} \dot{\psi}^{2}+V \\
& +\tilde{\lambda}^{2} e^{\frac{\psi}{\psi_{0}}}\left(-12 H_{a}^{3} \frac{\dot{\psi}}{\psi_{0}}+9 H_{a}^{2} \frac{\dot{\psi}^{2}}{\psi_{0}^{2}}-\frac{3}{4} \frac{\dot{\psi}^{4}}{\psi_{0}^{4}}\right), \\
p_{\psi}= & \left(\frac{1}{2} \dot{\psi}^{2}-V\right) \\
& +\tilde{\lambda}^{2} e^{\frac{\psi}{\psi_{0}}}\left(8 H_{a}\left(H_{a}^{2}+\dot{H}_{a}\right) \frac{\dot{\psi}}{\psi_{0}}+\left(H_{a}^{2}-2 \dot{H}_{a}\right) \frac{\dot{\psi}^{2}}{\psi_{0}^{2}}\right. \\
& \left.-2 H_{a} \frac{\dot{\psi}^{3}}{4 \psi_{0}^{3}}-\frac{\dot{\psi}^{4}}{\psi_{0}^{4}}+4\left(H_{a}^{2}-2 H_{a} \frac{\dot{\psi}^{2}}{\psi_{0}}\right) \frac{\ddot{\psi}}{\psi_{0}}\right) \\
V_{\mathrm{eff}}^{\prime}= & V^{\prime}+\tilde{\lambda}^{2} e^{\frac{\psi}{\psi_{0}}} \\
& \times\left(-\frac{12}{\psi_{0}} H_{a}^{2}\left(H_{a}^{2}+\dot{H}_{a}\right)-3 H_{a}^{2} \frac{\dot{\psi}^{2}}{\psi_{0}^{3}}-\frac{3}{4} \frac{\dot{\psi}^{4}}{\psi_{0}^{5}}\right. \\
& \left.+6 H_{a}\left(3 H_{a}^{2}+2 \dot{H}_{a}\right) \frac{\dot{\psi}^{2}}{\psi_{0}^{2}}+3\left(2 H_{a}^{2}-\frac{\dot{\psi}^{2}}{\psi_{0}^{2}}\right) \frac{\ddot{\psi}}{\psi_{0}^{2}}\right) .
\end{aligned}
$$

At the initial time, $t=t_{i}, \dot{\psi} / \psi_{i} \ll 0, V_{\text {eff }}^{\prime}$ can be approximated as

$V_{\mathrm{eff}}^{\prime} \sim V^{\prime}-\tilde{\lambda}^{2} e^{\frac{\psi}{\psi_{0}}} \frac{12}{\psi_{0}} H_{a}^{2}\left(H_{a}^{2}+\dot{H}_{a}\right)$.

Again, the effective force acting on the radion field is reduced at the initial time. Using the same method in the fivedimensional case, the decreasing of the effective force will lead to restoration of the stabilization mechanism. This analysis is confirmed by numerical simulation of the equations of motion in six-dimensional spacetime. In six-dimensional spacetime, we have nine degrees of freedom for the graviton. We choose this also as the number of degrees of freedom for the massless boson in the total Casimir energy density. The other numbers are obtained in the same way as discussed in the five-dimensional case. The number of degrees of freedom for the massless fermion, massive boson, and massive fermion can be chosen, respectively, as $N_{f}=\tilde{N}_{b}=\tilde{N}_{f}=$ 16. By using this particle spectrum, the total Casimir energy density in six-dimensional spacetime can be written as

$\rho_{\text {Cas }}^{(6)}=-7 \rho_{\text {Cas }}^{\text {massless }}+16 \rho_{\text {Cas }}^{\text {massive }}\left(m_{b}\right)-16 \rho_{\text {Cas }}^{\text {massive }}\left(m_{f}\right)$,

where the mass ratio is chosen as the lower bound, $\bar{\lambda}=0.456$. The range of the parameter $\lambda$ for restoring the stabilization mechanism is $0.61<\lambda<1.44$. The range is sensitive to the mass ratio and also depends on the initial value of $b(t)$. We note that, replacing the non-relativistic matter with radiation, the stabilization mechanism in six-dimensional spacetime still holds. The action in four-dimensional spacetime reduced from five-dimensional spacetime in Eq. (36) and six-dimensional spacetime in Eq. (44) can be considered as a modified gravity theory. The modification provides some corrections to Einstein gravity which can be constrained by experiments at the solar system scale, for example [96-98]. Note that the potential form in [96-98] is chosen to be an exponential function of the scalar field, since most of the investigations do not try to address the moduli stabilization problem. In order to constrain our theoretical parameters such as $\bar{\lambda}$ and $\lambda$, one may find some corrections of our model in the same manner as done in the literature but the potential form in our case comes from Casimir energy density. It is important to note that the allowed region of the parameters may not be consistent with the stability condition in order to avoid ghost degrees of freedom in the model [99-102]. We leave this investigation of our model including the constraints of the theoretical parameters for further work.

\section{Conclusions}

The concept of a Casimir dark energy model has been reviewed. The important idea of this model is that it is natural to interpret the Casimir energy emerging from the compactification of the extra dimensions as dark energy to drive the late-time accelerating universe [35]. However, this model of dark energy encounters the problem that the extra dimensions cannot be stabilized when non-relativistic matter is taken into account. One solution of this problem is adding an exotic field such as an aether field to the model [42]. However, the aether theory itself is not stable [45-48]. In this paper, we seek another solution by generalizing the Einstein 
gravity theory to Einstein-Gauss-Bonnet (EGB) gravity theory. It is worthwhile to investigate EGB theory since it is a generalization of Einstein gravity in higher-dimensional spacetime which keeps second order derivatives in the equations of motion and satisfies the conservation equation of the matter field. It is also compatible with the low-energy effective field theory of string theory [70,71]. The results of our investigation in five-dimensional spacetime showed that the radius of the extra dimension can be stabilized when the Gauss-Bonnet (GB) term and non-relativistic matter are taken into account. The extension by including radiation into the model was also investigated and the result showed that the stabilization mechanism still holds. We used the radion picture in four-dimensional spacetime to analyze how the GB term can provide the stabilization mechanism. It was found that the Gauss-Bonnet contribution effectively reduces the slope of the radion potential at the initial time corresponding to a reduction of the magnitude of the force acting on the radion field. Therefore, the radion field slowly rolls down and does not pass the point at which the minimum of the potential will arise before the minimum has formed so that the stabilization mechanism is eventually restored. We also investigated this behavior in a six-dimensional spacetime. The extra dimensions can be stabilized in the same manner as in the five-dimensional case. The stability of the model by itself is an important issue for investigation. The effective four-dimensional GB theory with an exponential potential and without terms corresponding to a nonminimal coupling to gravity are found to be unstable due to the tachyonic instability $[102,103]$. The extension of the model including nonminimal coupling terms, without a potential, implies that the model is stable but the matter phase is absent in the history of the universe $[94,95]$. Adding the potential term corresponding to the Casimir energy may provide this matter phase. We leave this investigation, including the constraints on the model, parameters for further work. Finally, the interplay between the Gauss-Bonnet term and the dynamical radion field in our model may shed some light on the connection between modified gravity theories and the fundamental high-energy theories which require higher-dimensional spacetimes.

Acknowledgments The author would like to thank the String Theory and Supergravity Group at Chulalongkorn University for hospitality during the progress of this work. He is also grateful to Parinya Karndumri and to Antonio De Felice for helpful conversations and comments regrading the manuscript. It is a great pleasure to thank the referee for useful comments. He also thanks Matthew James Lake for reading through the manuscript and correcting some grammatical errors. This work is supported by the Naresuan University Research Fund through grant R2556C042.

Open Access This article is distributed under the terms of the Creative Commons Attribution License which permits any use, distribution, and reproduction in any medium, provided the original author(s) and the source are credited.

Funded by $\mathrm{SCOAP}^{3}$ / License Version CC BY 4.0.

\section{Appendix A: Diagonal Kaluza-Klein compactification}

In this appendix we review a special case of generalized Kaluza-Klein compactification [89]. We restrict our attention to diagonal metrics for the internal extra dimensions for simplicity. The metric can be written as

$$
\begin{aligned}
d s^{2} & =g_{A B} d x^{A} d x^{B}, \\
& =e^{2 \alpha \phi} \bar{g}_{\mu \nu} d x^{\mu} d x^{\nu}+e^{2 \beta \phi} \tilde{g}_{a b} d y^{a} d y^{b},
\end{aligned}
$$

where the indices $A, B, \ldots$ run over the whole $\mathrm{D}$-dimensional spacetime, the indices $\mu, v, \ldots$ run over the $(3+1)$ dimensional spacetime and the indices $a, b, \ldots$ run over the internal space of $n$ dimensions. For simplicity, we assume that $\bar{g}_{\mu \nu}$ and $\tilde{g}_{a b}$ are diagonal and $\phi$ depends only on the external spacetime coordinates, $\phi=\phi\left(x^{\mu}\right) . \alpha$ and $\beta$ are parameters which we will choose later in order to compare the results with four-dimensional theory. Note that the scalar field $\phi$ is not an external field. It is a scalar degree of freedom of the metric $\tilde{g}_{a b}$ such that $\tilde{g}_{a b} \rightarrow e^{2 \beta \phi} \tilde{g}_{a b}$. This leads to the transformation of the determinant of the metric as $\sqrt{\operatorname{det}\left(\tilde{g}_{a b}\right)} \rightarrow e^{n \beta \phi} \sqrt{\operatorname{det}\left(\tilde{g}_{a b}\right)}$, where the quantity $\sqrt{\operatorname{det}\left(\tilde{g}_{a b}\right)}$ on the right hand side of the arrow needs to be a constant. In our derivation of the Kaluza-Klein reduction, we show the explicit expression including $\sqrt{\tilde{g}}$ even though it is just a constant which can be absorbed into the scalar field $\phi$ by rescaling. In the standard procedure of Kaluza-Klein reduction [104], we do not need to put the conformal factor $e^{2 \alpha \phi}$ over the metric $\bar{g}_{\mu \nu}$ and the parameter $\beta$ can be set as a constant before being absorbed into the field $\phi$. Using this procedure, the quantity $\sqrt{-g} R$ in D-dimensional spacetime will be related to the quantity $\sqrt{-\bar{g}} \bar{R}$ in (1+3)-dimensional spacetime via $\sqrt{-g} R=\sqrt{-\bar{g}} e^{n \phi} \bar{R}+\cdots$, where the ellipsis denotes other terms. In order to obtain the theory in the Einstein frame, we have to use a conformal transformation to get rid of the factor $e^{n \phi}$ over the Ricci scalar in a noncompact spacetime. Therefore, it is convenient to put the conformal factor to the metric $\bar{g}_{\mu \nu}$ at the beginning time as shown in above equation. In this sense, one can find the relation between the parameters $\alpha$ and $\beta$ to get rid of the overall factor of $\bar{R}$, as we will show below. Using this ansatz and the calculation as in standard procedure of Kaluza-Klein reduction [104], the Ricci scalar can be written as

$$
\begin{aligned}
\sqrt{-g} R= & \sqrt{-\bar{g}} \sqrt{\tilde{g}} e^{(2 \alpha+n \beta) \phi}\left(e^{2(\alpha-\beta)} \tilde{R}-2(3 \alpha+n \beta) \square \phi\right. \\
& \left.+\bar{R}-\left(6 \alpha^{2}+n(n+1) \beta^{2}+4 n \alpha \beta\right)(\partial \phi)^{2}\right) .
\end{aligned}
$$


We note that the quantities with a "bar", $\bar{X}$, stand for quantities in $(3+1)$-external spacetime and the quantities with a "tilde", $\tilde{X}$, stand for quantities in $n$-internal space. In order to get our usual $(3+1)$-dimensional spacetime, one can set $\beta=-2 \alpha / n$ to get rid of the overall factor of $\bar{R}$. Substituting $\beta$ back into Eq. (A.2), we have

$$
\begin{aligned}
\sqrt{-g} R= & \sqrt{-\bar{g}} \sqrt{\tilde{g}}\left(\bar{R}+e^{\frac{2}{n}(n+2) \alpha \phi} \tilde{R}\right. \\
& \left.-2 \alpha \square \phi-\frac{2}{n}(n+2) \alpha^{2}(\partial \phi)^{2}\right) .
\end{aligned}
$$

For the Gauss-Bonnet term, the calculation is very lengthy but straightforward. After integrating by parts and then setting the total derivative terms to zero, the Gauss-Bonnet term can be written as

$$
\begin{aligned}
\sqrt{-g} \mathcal{G}= & \sqrt{-\bar{g}} \sqrt{\tilde{g}}\left[e ^ { n \beta \phi } \left(\overline{\mathcal{G}}-4 f_{1} \bar{G}^{\mu \nu} \partial_{\mu} \phi \partial_{\nu} \phi\right.\right. \\
& \left.-2 f_{2}(\partial \phi)^{2} \square \phi-f_{3}(\partial \phi)^{2}(\partial \phi)^{2}\right) \\
& +e^{(4 \alpha+(n-4) \beta) \phi} \tilde{\mathcal{G}} \\
& \left.+e^{(2 \alpha+(n-2) \beta) \phi} \tilde{R}\left(\bar{R}+f_{4}(\partial \phi)^{2}\right)\right],
\end{aligned}
$$

where

$$
\begin{aligned}
f_{1}= & 2 n \alpha \beta+n(n-1) \beta^{2}, \\
f_{2}= & 6 n \alpha^{2} \beta+6 n(n-1) \alpha \beta^{2}+n(n-1)(n-2) \beta^{3}, \\
f_{3}= & 8 n \alpha^{3} \beta+4 n(4 n-3) \alpha^{2} \beta^{2} \\
& +8 n(n-1)^{2} \alpha \beta^{3}+8 n(n-1)^{2}(n-2) \beta^{4}, \\
f_{4}= & 6 \alpha^{2}+6(n-2) \alpha \beta+(n-2)(n-3) \beta^{2} .
\end{aligned}
$$

This reduction of the Gauss-Bonnet term is a special case of the result given in Ref. [89]. Note that the reduction in [89] is performed from $(1+p+n)$-dimensional spacetime to $(1+p)$-dimensional spacetime. If we choose the parameter as $\beta=-2 \alpha / n$, the Gauss-Bonnet term becomes

$$
\begin{aligned}
\sqrt{-g} \mathcal{G}= & \sqrt{-\bar{g}} \sqrt{\tilde{g}}\left[e ^ { - 2 \alpha \phi } \left(\overline{\mathcal{G}}-4 f_{1} \bar{G}^{\mu \nu} \partial_{\mu} \phi \partial_{\nu} \phi\right.\right. \\
& \left.-2 f_{2}(\partial \phi)^{2} \square \phi-f_{3}(\partial \phi)^{2}(\partial \phi)^{2}\right) \\
& \left.+e^{4 \alpha \phi / n} \tilde{R}\left(\bar{R}+f_{4}(\partial \phi)^{2}\right)+e^{2(n-4) \alpha \phi / n} \tilde{\mathcal{G}}\right] .
\end{aligned}
$$

For the cosmological constant and matter terms, the reduction factor comes from $\sqrt{-g}$. These terms can be written as

$$
\begin{aligned}
\sqrt{-g} \Lambda & =\sqrt{-\bar{g}} \sqrt{\tilde{g}} e^{(4 \alpha+n \beta) \phi} \Lambda \\
& =\sqrt{-\bar{g}} \sqrt{\tilde{g}} e^{2 \alpha \phi} \lambda^{-2}, \\
\sqrt{-g} L_{m} & =\sqrt{-\bar{g}} \sqrt{\tilde{g}} e^{(4 \alpha+n \beta) \phi} L_{m}
\end{aligned}
$$

$$
=\sqrt{-\bar{g}} \sqrt{\tilde{g}} e^{2 \alpha \phi} L_{m} .
$$

We assume that $\tilde{g}_{\mu \nu}$ is a Euclidean metric. Note that we also use this assumption for calculating the Casimir energy density. Hence, we can integrate out the extra dimension coordinates. To obtain the Newton gravitational constant in fourdimensional spacetime, the mass scale must relate to the Planck mass as follows:

$M_{P l}^{2}=M_{*}^{n+2} \int d^{n} y \sqrt{\tilde{g}}=M_{*}^{n+2}(2 \pi)^{n}$.

Since $\tilde{g}_{\mu \nu}$ is a Euclidean metric, $\tilde{R}=\tilde{\mathcal{G}}=0$, the EGB action can be written as

$$
\begin{aligned}
S_{E G B}= & \int d^{4} x \sqrt{-\bar{g}} \frac{M_{P l}^{2}}{2}\left[\lambda ^ { 2 } e ^ { - 2 \alpha \phi } \left(\overline{\mathcal{G}}-4 f_{1} \bar{G}^{\mu \nu} \partial_{\mu} \phi \partial_{\nu} \phi\right.\right. \\
& \left.-2 f_{2}(\partial \phi)^{2} \square \phi-f_{3}(\partial \phi)^{2}(\partial \phi)^{2}\right)-2 \Lambda e^{2 \alpha \phi} \\
& \left.+\left(\bar{R}-\frac{2}{n}(n+2) \alpha^{2}(\partial \phi)^{2}\right)+\frac{2(2 \pi)^{n}}{M_{P l}^{2}} e^{2 \alpha \phi} L_{m}\right],
\end{aligned}
$$

where we have set $\alpha_{0}=-2, \alpha_{1}=\alpha_{2}=1$. In order to obtain the canonical form of the scalar field, one has to set

$\alpha=-\sqrt{\frac{n}{4(n+2)}}$,

and then the EGB action becomes

$$
\begin{aligned}
S_{E G B}= & \int d^{4} x \sqrt{-\bar{g}} \frac{M_{P l}^{2}}{2}\left[\lambda ^ { 2 } e ^ { \sqrt { \frac { n } { ( n + 2 ) } } \phi } \left(\overline{\mathcal{G}}-4 f_{1} \bar{G}^{\mu \nu} \partial_{\mu} \phi \partial_{\nu} \phi\right.\right. \\
& \left.-2 f_{2}(\partial \phi)^{2} \square \phi-f_{3}(\partial \phi)^{2}(\partial \phi)^{2}\right)-2 \Lambda e^{-\sqrt{\frac{n}{(n+2)}} \phi} \\
& \left.+\left(\bar{R}-\frac{1}{2}(\partial \phi)^{2}\right)+\frac{2(2 \pi)^{n}}{M_{P l}^{2}} e^{-\sqrt{\frac{n}{(n+2)}} \phi} L_{m}\right] .
\end{aligned}
$$

\section{Appendix B: Pressure regularization}

The pressure of the Casimir vacuum energy compatible with the metric in Eq. (1) can be defined as

$$
\begin{aligned}
& p_{a} \equiv-\frac{\partial}{\partial V_{a}}\left(\rho_{\text {Cas }} V_{a}\right)=-\rho_{\text {Cas }}, \\
& p_{b} \equiv-\frac{\partial}{\partial V_{b}}\left(\rho_{\text {Cas }} V_{b}\right)=-\frac{b}{V_{b}} \frac{\partial}{\partial b}\left(\frac{\widehat{E}_{\text {Cas }}}{L^{3}}\right),
\end{aligned}
$$

where $V_{a} \propto a^{3}, V_{b} \propto b$ and $\rho_{\text {Cas }}=\widehat{E}_{\text {Cas }} /\left(L^{3} V_{b}\right)$. These definitions are valid for any kinds of perfect fluid obeying the equation of state $p=w \rho$. One can check this statement by substituting the energy density of perfect fluid in a non-compact spacetime, $\rho=\rho_{0} a^{-(1+w) / 3}$, into Eq. (B.16). These forms of the pressure also satisfy the conservation 
equation of the energy-momentum tensor in Eq. (13). Note that $p_{a}=-\rho_{\text {Cas }}$ in Eq. (B.16) is obtained by the fact that $\widehat{E}_{\text {Cas }}$ does not depend on $a$ and then $\rho_{\text {Cas }}$ is independent of $V_{a}$. In order to find the expression of $p_{b}$, let us consider the quantity $\widehat{E}_{\text {Cas }} / L^{3}$ where $\widehat{E}_{\text {Cas }}$ is defined in Eq. (4). By using the relation $\int d^{n} k f(k)=2 \pi^{n / 2} /(\Gamma(n / 2)) \int d k k^{n-1} f(k)$, where $\Gamma(x)$ is the gamma function, one obtains

$$
\frac{\widehat{E}_{\mathrm{Cas}}}{L^{3}}=\frac{\pi^{-3 / 2}}{2^{4} \Gamma(3 / 2)} \int d k k^{2} \sum_{\tilde{n}} \sqrt{k^{2}+m^{2}+\frac{\tilde{n}^{2}}{b^{2}}},
$$

Using the integral representation of the gamma function and its properties, one obtains

$$
\frac{\widehat{E}_{\mathrm{Cas}}}{L^{3}}=\frac{\Gamma(-2) \pi^{5 / 2}}{(2 \pi b)^{4} \Gamma(-1 / 2)} \sum_{\tilde{n}}\left((m b)^{2}+\tilde{n}^{2}\right)^{2} .
$$

Note that taking the derivative with respect to $b$ before or after rearrange this expression yields the same result, since both integral representation and properties of the gamma function used for the above procedure do not depend on the variable b.

For the massless case, it is obvious that the summation can be written in the form of zeta function which is independent of $b$. Using relation of zeta function, $\Gamma(s / 2) \zeta(s) \pi^{-s / 2}=$ $\Gamma((1-s) / 2) \zeta(1-s) \pi^{-(1-s) / 2}$, one obtains

$\frac{\widehat{E}_{\mathrm{Cas}}^{(\text {massless })}}{L^{3}}=\frac{2 \Gamma(5 / 2) \zeta(5)}{\Gamma(-1 / 2)(2 \pi b)^{4} \pi^{2}}$.

Substituting this quantity into Eq. (B.17), the vacuum pressure contributed from compact direction can be written as

$p_{b}^{m l}=4 \frac{2 \Gamma(5 / 2) \zeta(5)}{\Gamma(-1 / 2)(2 \pi b)^{5} \pi^{2}}=4 \rho_{\mathrm{Cas}}^{\text {massless }}$.

For the massive case, one can find finite part of the pressure by using Chowla-Selberg zeta function. The result of regularization will be the same whether we take the derivative first and then regularize or regularize first and then take the derivative. This will be shown below. For the first option, substituting Eq. (B.19) into Eq. (B.17), one obtains

$$
\begin{aligned}
& p_{b}^{m s}=\frac{b \Gamma(-2) \pi^{2}}{2 V_{b}(2 \pi b)^{4}} \\
& \times\left(\frac{-4}{b} \sum_{\tilde{n}}\left((m b)^{2}+\tilde{n}^{2}\right)^{2}+2 m^{2} b \sum_{\tilde{n}}\left((m b)^{2}+\tilde{n}^{2}\right)\right) .
\end{aligned}
$$

Using the Chowla-Selberg zeta function and keeping only the finite part, the summation can be expressed as

$$
\sum_{l}\left(q^{2}+l^{2}\right)^{-s}=\frac{4 \pi^{s} q^{1 / 2-s}}{\Gamma(s)} \sum_{\tilde{n}=1}^{\infty} \tilde{n}^{s-1 / 2} K_{1 / 2-s}(2 \pi q \tilde{n}) .
$$

Substituting this expression into Eq. (B.22), one obtains

$p_{b}^{m s}=4 \rho_{\text {Cas }}^{\text {massive }}-\frac{2 \pi(m b)^{7 / 2}}{(2 \pi b)^{5}} \sum_{\tilde{n}=1}^{\infty} \tilde{n}^{-3 / 2} K_{3 / 2}(2 \pi m b \tilde{n})$.

For the second option, one can use the regularized energy density in Eq. (7) and then take the derivative through Eq. (B.17) as follows:

$$
\begin{aligned}
p_{b}^{m s}= & \frac{2 b}{V_{b}} \frac{\partial}{\partial b}\left(\frac{(m b)^{5 / 2}}{(2 \pi b)^{4}} \sum_{\tilde{n}=1}^{\infty} \tilde{n}^{-5 / 2} K_{5 / 2}(2 \pi m b \tilde{n})\right) \\
= & 4 \rho_{\text {Cass }}^{\text {massive }}+\frac{2 b}{V_{b}(2 \pi b)^{4}} \frac{\partial}{\partial b} \\
& \times\left((m b)^{5 / 2} \sum_{\tilde{n}=1}^{\infty} \tilde{n}^{-5 / 2} K_{5 / 2}(2 \pi m b \tilde{n})\right) \\
= & 4 \rho_{\text {Cas }}^{\text {massive }}-\frac{2 \pi(m b)^{7 / 2}}{(2 \pi b)^{5}} \sum_{\tilde{n}=1}^{\infty} \tilde{n}^{-3 / 2} K_{3 / 2}(2 \pi m b \tilde{n})
\end{aligned}
$$

where we have used the differential equation of the modified Bessel function as

$\frac{d}{d x}\left(x^{v} K_{v}(x)\right)=-x^{v} K_{v-1}(x)$.

Since the total Casimir energy density is a particular choice of a linear combination of massless, massive boson, and massive fermion Casimir energy density expressed in Eq. (8), the total Casimir pressure can be written as

$$
\begin{aligned}
p_{a}= & -\rho_{\text {Cas }}=-3 \rho_{\text {Cas }}^{\text {massless }}+8 \rho_{\text {Cas }}^{\text {massive }}\left(m_{b}\right) \\
& -8 \rho_{\text {Cas }}^{\text {massive }}\left(m_{f}\right), \\
p_{b}= & -3 p_{b}^{m l}+8 p_{b}^{m s}\left(m_{b}\right)-8 p_{b}^{m s}\left(m_{f}\right), \\
= & 4 \rho_{\text {Cas }}-\frac{16 \pi}{(2 \pi b)^{5}} \sum_{\tilde{n}=1}^{\infty} \tilde{n}^{-3 / 2} \\
& \times\left(\frac{K_{3 / 2}\left(2 \pi m_{b} b \tilde{n}\right)}{\left(m_{b} b\right)^{-7 / 2}}-\frac{K_{3 / 2}\left(2 \pi m_{f} b \tilde{n}\right)}{\left(m_{f} b\right)^{-7 / 2}}\right),
\end{aligned}
$$

where the explicit dependence of $\rho_{\text {Cas }}$ on $b$ can be expressed as

$$
\begin{aligned}
\rho_{\mathrm{Cas}}= & -\frac{1}{(2 \pi b)^{5}}\left(-3 \frac{\Gamma\left(\frac{5}{2}\right) \zeta(5)}{\pi^{5 / 2}}+16 \sum_{\tilde{n}=1}^{\infty} \tilde{n}^{-5 / 2}\right. \\
& \left.\times\left(\frac{K_{5 / 2}\left(2 \pi m_{b} b \tilde{n}\right)}{\left(m_{b} b\right)^{-5 / 2}}-\frac{K_{5 / 2}\left(2 \pi m_{f} b \tilde{n}\right)}{\left(m_{f} b\right)^{-5 / 2}}\right)\right) .
\end{aligned}
$$

\section{References}

1. A.G. Riess et al., Supernova Search Team Collaboration. Astron. J. 116, 1009 (1998) 
2. S. Perlmutter et al., Supernova Cosmology Project Collaboration. Astrophys. J. 517, 565 (1999)

3. D.N. Spergel et al., WMAP Collaboration. Astrophys. J. Suppl. 148, 175 (2003)

4. D.J. Eisenstein et al., SDSS Collaboration. Astrophys. J. 633, 560 (2005)

5. P.A.R. Ade et al. [Planck Collaboration], arXiv:1303.5076 [astroph.CO]

6. S. Weinberg, Rev. Mod. Phys. 61, 1 (1989)

7. Y.B. Zeldovich, JETP Lett. 6, 316 (1967)

8. Y.B. Zeldovich, Pisma. Zh. Eksp. Teor. Fiz. 6, 883 (1967)

9. Y.B. Zeldovich, Sov. Phys. Usp. 11, 381 (1968)

10. Y.B. Zeldovich, Gen. Rel. Grav. 40, 1557 (2008)

11. V. Sahni, A.A. Starobinsky, Int. J. Mod. Phys. D 9, 373 (2000)

12. E.J. Copeland, M. Sami, S. Tsujikawa, Int. J. Mod. Phys. D 15, $1753(2006)$

13. C. Wetterich, Nucl. Phys. B 302, 668 (1988)

14. B. Ratra, P.J.E. Peebles, Phys. Rev. D 37, 3406 (1988)

15. C. Armendariz-Picon, V.F. Mukhanov, P.J. Steinhardt, Phys. Rev. Lett. 85, 4438 (2000)

16. C. Armendariz-Picon, V.F. Mukhanov, P.J. Steinhardt, Phys. Rev. D 63, 103510 (2001)

17. T. Chiba, T. Okabe, M. Yamaguchi, Phys. Rev. D 62, 023511 (2000)

18. A. Nicolis, R. Rattazzi, E. Trincherini, Phys. Rev. D 79, 064036 (2009)

19. C. Deffayet, G. Esposito-Farese, A. Vikman, Phys. Rev. D 79, 084003 (2009)

20. C. Deffayet, S. Deser, G. Esposito-Farese, Phys. Rev. D 80, 064015 (2009)

21. V.V. Kiselev, Class. Quant. Grav. 21, 3323 (2004)

22. C. Armendariz-Picon, JCAP 0407, 007 (2004)

23. C. Germani, A. Kehagias, JCAP 0903, 028 (2009)

24. T.S. Koivisto, D.F. Mota, C. Pitrou, JHEP 0909, 092 (2009)

25. M. Li, Phys. Lett. B 603, 1 (2004)

26. S. Capozziello, S. Carloni, A. Troisi, Recent Res. Dev. Astron. Astrophys. 1, 625 (2003)

27. S.M. Carroll, V. Duvvuri, M. Trodden, M.S. Turner, Phys. Rev. D 70, 043528 (2004)

28. W. Hu, I. Sawicki, Phys. Rev. D 76, 064004 (2007)

29. S.A. Appleby, R.A. Battye, Phys. Lett. B 654, 7 (2007)

30. A.A. Starobinsky, JETP Lett. 86, 157 (2007)

31. S. 'i. Nojiri, S.D. Odintsov, Phys. Lett. B 631, 1 (2005)

32. C. de Rham, G. Gabadadze, Phys. Rev. D 82, 044020 (2010)

33. C. de Rham, G. Gabadadze, A.J. Tolley, Phys. Rev. Lett. 106, 231101 (2011)

34. E. Ponton, E. Poppitz, JHEP 0106, 019 (2001)

35. B.R. Greene, J. Levin, JHEP 0711, 096 (2007)

36. R.H. Brandenberger, Prog. Theor. Phys. Suppl. 163, 358 (2006)

37. H. Kodama, K. Uzawa, JHEP 0603, 053 (2006)

38. H. Kodama, K. Uzawa, hep-th/0601100

39. G. Shiu, Y. Sumitomo, JHEP 1109, 052 (2011)

40. A. Flachi, M. Minamitsuji, K. Uzawa, JHEP 1308, 073 (2013)

41. P. Burikham, A. Chatrabhuti, P. Patcharamaneepakorn, K. Pimsamarn, JHEP 0807, 013 (2008)

42. A. Chatrabhuti, P. Patcharamaneepakorn, P. Wongjun, JHEP 0908, 019 (2009)

43. T.G. Rizzo, JHEP 0509, 036 (2005)

44. S.M. Carroll, H. Tam, Phys. Rev. D 78, 044047 (2008)

45. S.M. Carroll, T.R. Dulaney, M.I. Gresham, H. Tam, Phys. Rev. D 79, 065011 (2009)

46. B. Himmetoglu, C.R. Contaldi, M. Peloso, Phys. Rev. D 79, 063517 (2009)

47. B. Himmetoglu, C.R. Contaldi, M. Peloso, Phys. Rev. Lett. 102, 111301 (2009)
48. B. Himmetoglu, C.R. Contaldi, M. Peloso, Phys. Rev. D 80, 123530 (2009)

49. M. Williams, C.P. Burgess, L. van Nierop, A. Salvio, JHEP 1301, $102(2013)$

50. C.P. Burgess, L. van Nierop, S. Parameswaran, A. Salvio, M. Williams, JHEP 1302, 120 (2013)

51. A. Salvio, J. Phys. Conf. Ser. 437, 012004 (2013)

52. L. Randall, R. Sundrum, Phys. Rev. Lett. 83, 3370 (1999)

53. L. Randall, R. Sundrum, Phys. Rev. Lett. 83, 4690 (1999)

54. R. Maartens, K. Koyama, Living Rev. Rel. 13, 5 (2010)

55. W.D. Goldberger, M.B. Wise, Phys. Rev. Lett. 83, 4922 (1999)

56. C. Csaki, M. Graesser, L. Randall, J. Terning, Phys. Rev. D 62, 045015 (2000)

57. T. Tanaka, X. Montes, Nucl. Phys. B 582, 259 (2000)

58. J. Garriga, T. Tanaka, Phys. Rev. Lett. 84, 2778 (2000)

59. J. Garriga, O. Pujolas, T. Tanaka, Nucl. Phys. B 605, 192 (2001)

60. J. Garriga, O. Pujolas, T. Tanaka, Nucl. Phys. B 655, 127 (2003)

61. A. Flachi, I.G. Moss, D.J. Toms, Phys. Lett. B 518, 153 (2001)

62. A. Flachi, I.G. Moss, D.J. Toms, Phys. Rev. D 64, 105029 (2001)

63. A. Flachi, J. Garriga, O. Pujolas, T. Tanaka, JHEP 0308, 053 (2003)

64. J. Lesgourgues, L. Sorbo, Phys. Rev. D 69, 084010 (2004)

65. D. Konikowska, M. Olechowski, M.G. Schmidt, Phys. Rev. D 73, 105018 (2006)

66. G.L. Alberghi, A. Tronconi, Phys. Rev. D 73, 027702 (2006)

67. C. Lanczos, Ann. Math. 39, 842 (1938)

68. D. Lovelock, J. Math. Phys. 12, 498 (1971)

69. B. Zumino, Phys. Rept. 137, 109 (1986)

70. I. Antoniadis, J. Rizos, K. Tamvakis, Nucl. Phys. B 415, 497 (1994)

71. B.A. Campbell, M.J. Duncan, N. Kaloper, K.A. Olive, Nucl. Phys. B 351, 778 (1991)

72. T. Kaluza, Sitzungsber. Preuss. Akad. Wiss. Berlin (Math. Phys.) 1921, 966 (1921)

73. O. Klein, Z. Phys. 37, 895 (1926)

74. O. Klein, Surveys High Energ. Phys. 5, 241 (1986)

75. H.B.G. Casimir, Indag. Math. 10, 261 (1948)

76. H.B.G. Casimir, Kon. Ned. Akad. Wetensch. Proc. 51, 793 (1948)

77. H.B.G. Casimir, Front. Phys. 65, 342 (1987)

78. H.B.G. Casimir, Kon. Ned. Akad. Wetensch. Proc. 100N3-4, 61 (1997)

79. M.J. Sparnaay, Physica 24, 751 (1958)

80. S.K. Lamoreaux, Phys. Rev. Lett. 78, 5 (1997) [Erratum-ibid. 81, 5475 (1998)]

81. E. Elizalde, S.D. Odintsov, A. Romeo, A.A. Bytsenko, S. Zerbini, Zeta Regularization Techniques with Applications (World Scientific, Singapore, 1994)

82. T. Appelquist, A. Chodos, Phys. Rev. D 28, 772 (1983)

83. M. Maggiore, L. Hollenstein, M. Jaccard, E. Mitsou, Phys. Lett. B 704, 102 (2011)

84. M. Maggiore, Phys. Rev. D 83, 063514 (2011)

85. M. Asorey, P.M. Lavrov, B.J. Ribeiro, I.L. Shapiro, Phys. Rev. D 85, 104001 (2012). arXiv:1202.4235 [hep-th]

86. P.G.O. Freund, Nucl. Phys. B 209, 146 (1982)

87. N. Deruelle, J. Madore, gr-qc/0305004

88. C. Charmousis, Lect. Notes Phys. 769, 299 (2009)

89. C. Charmousis, B. Gouteraux, E. Kiritsis, JHEP 1209, 011 (2012)

90. S. Kawai, M.-a. Sakagami, J. Soda. Phys. Lett. B 437, 284 (1998)

91. S. Kawai, J. Soda, Phys. Lett. B 460, 41 (1999)

92. S. Kawai, J. Soda, Phys. Rev. D 59, 063506 (1999)

93. E.J. Kim, S. Kawai, Phys. Rev. D 87, 083517 (2013)

94. R. Gannouji, M. Sami, Phys. Rev. D 85, 024019 (2012)

95. T. Kobayashi, M. Yamaguchi, J. 'i. Yokoyama, Prog. Theor. Phys. 126, 511 (2011)

96. L. Amendola, C. Charmousis, S.C. Davis, JCAP 0612, 020 (2006) 
97. T. Koivisto, D.F. Mota, Phys. Lett. B 644, 104 (2007)

98. L. Amendola, C. Charmousis, S.C. Davis, JCAP 0710, 004 (2007)

99. K.S. Stelle, Gen. Rel. Grav. 9, 353 (1978)

100. N.H. Barth, S.M. Christensen, Phys. Rev. D 28, 1876 (1983)

101. A. De Felice, M. Hindmarsh, M. Trodden, JCAP 0608, 005 (2006)
102. G. Calcagni, B. de Carlos, A. De Felice, Nucl. Phys. B 752, 404 (2006)

103. S. Tsujikawa, M. Sami, JCAP 0701, 006 (2007)

104. S.M. Carroll, Spacetime and Geometry: An introduction to General Relativity (Addison Wesley Press, Sanfrancisco, 2004) 